\title{
A Comparative Study of Serum Alt \&Ast Level And Ast/Alt Ratio In Alcoholic And Non-Alcoholic Acute Uncomplicated Falciparum Malaria Without Clinical Jaundice.
}

\author{
Dr. Divakar Kumar ${ }^{1}$, Dr. C. B. Sharma ${ }^{2}$ \\ ${ }^{1}$ Senior Resident, Department of Medicine, RIMS, Ranchi \\ ${ }^{2}$ Associate Professor, Department of Medicine, RIMS, Ranchi
}

\begin{abstract}
Serum alanine aminotransferase (ALT) and aspartate aminotransfarese levels and AST/ALT ratio in 28 alcoholic and 26 nonalcoholic cases suffering from acute uncomplicated anicteric plasmodium falciparum malaria, age ranging from 18-70 years of both sexes. No. Of males and females were 18 and 10 in alcoholic uncomplicated falciparum malaria and $14 \& 12$ in non-alcoholic uncomplicated falciparum malaria cases respectively.

Cases for study were selected by random sampling among the patients suffering from fever admitted in department of medicine, rajendra institute of medical sciences, ranchi, who were subsequently proved by clinical and laboratory assessment to be cases of acute uncomplicated anicteric falciparum malaria and not associated with any other systemic and organic involvements.

Cases were divided in two groups comprising both males and female, alcoholics and non alcoholics on the basis of history of alcohol intake.

Serum ALT \&AST levels in all the cases selected for study were estimated once at admission.

The mean serum ALT\&AST levels were found to increase significantly in both groups but ALT\&AST levels were greater in alcoholic patients than in non alcoholic patients.

The mean AST/ALT ratio was found to be more than one in both groups but it was marginally more in alcoholic patients and the AST/ALT ratio found in both the studygroups would have been more than the commonly encountered chronic liver diseases like non-alcoholic steatohepatitis, chronic hepatitis $c$ and chronic hepatitis $b$ as per the review of previous reports and the ratio was less than the patients of denovo alcoholic liver disease.

So the conclusion drawn was mean serum ALT\&AST level and AST/ALT ratio were higher in alcoholic patients in comparison to nonalcoholic patient emphasizing the predictive value for the future hepatic dysfunction and to start the adequate and aggressive management at the earliest to prevent the further complication.

The AST/ALT ratio >1 reported previously to be highly specific for the diagnosis of alcoholic liver disease only but the present study confirms that the AST/ALT ratio as noninvasive means of assessing liver disease must be done with caution in tropical area where falciparum malaria fever have been amongst the leading causes of hospitalisation.
\end{abstract}

\section{Introduction}

The transaminases are two closely related enzymes of clinical importance particularly in the assessment of liver function and injury. Amongst the duo AST is known to exist in two electrophoretically distinct forms, a cationic isoenzyme associated with the mitochondria and the anionic isoenzyme associated with cytoplasm. Tissue levels of AST are highest in the heart \& liver . significant amount are also found in skeletal muscle, kidney with lower levels in pancreas, spleen and lung. Low levels of AST are also found in erythrocytes. ALT is present in varying concentration in the liver,heart, skeletal muscle, kidney, pancreas, spleen,lung and red blood cells. Both enzymes increase in many disorders related to the liver damage and hence they have been proven to be sensitive indicator of liver cell injury. In particular patient with alcoholic liver disesase and malarial hepatitis present with marked increase in serum levels of both AST\&ALT. ALT is more elevated than AST in various necroinflammatory condition of the liver reflecting its greater efficiency as a liver disesase marker. Serum AST levels are found to increase in myocardial infarction, muscle diseases and haemolytic anaemia. Other reported causes of elevated serumaminotransferase levels include alcohol abuse,medications, autoimmune hepatitis, non-alcoholic steatohepatitis,haemochromatosis, Wilson's disease and alpha 1 antitrypsin deficiency. However it is not only diseases affecting the liver that are associated with elevated serum aminotransferases, other causesinclude occult celiac sprue, muscular dystrophy, acute appendicitis, obesity. Because of their usefulness as serummarker of liver disease the AST,ALT level and AST/ALT ratio has been postulatedto be good indicator of hepatic disease in adult. AST/ALT ratio has been used to distinguish cirrhotic from noncirrhotic patients and the patientswith non alcoholic steatohepatitis from those with alcoholic liver disease. In this study we assayed for the serum levels of both AST \&ALT in alcoholic and non acholic adult 
presenting with acute uncomplicated anicteric falciparum malaria and compared the AST/ALT ratio obtained with AST/ALT ratio reported for other diseases.

\section{Material And Methods}

Study was done on indoor patients admitted in the department of medicine at RIMS,ranchi from 1aug2014-30sep. 2014, beause in these months of year the incidence of falciparum malaria is at its highest peak due to higest average rainfall occuring in thse months.Patient selection was done by simple random sampling of individuals admitted in indoor with a history of fever and some cases associated with chill \&rigor who were confirmed to be infected with falciparum malaria parasite by microscopic examination of giemsa stained thin blood slides and optimal test. Based on the following selection criteria and h/o alcohol intake patients were grouped in alcoholic and non-alcoholic. Alcoholic group included 28 males \&10 females, non-alcoholic group included 14 males \&12 females. The age range of patients was 18-70 years comprising both sexes. other disorders like anaemia, cirrhosis of liver, viral hepatitis ,autoimmune hepatitis, a1 antitrypsin deficiency ,wilson's disease, haemochromatosis, kidney disorders were excluded from study by the process of clinical exam., Routine lab. Inv. like CBC, LFT,RFT,RBS,R/E of urine, Hbsag,HIV,X-ray chest PA view, UsG abd and as per noted some special investigation. In our study patient's having serum bilirubin $>2 \mathrm{mg} / \mathrm{dl}$ were excluded from study.

Serum venous blood were obtained from each of the patient by venepuncture of the ante cubital vein using sterile needle and syringe. The blood sample were then transferred into clean, sterile tube and allowed to clot. Each clotted sample was centrifuged for $10 \mathrm{~min}$. to obtain the sera. Enzymes assay was carried out within $24 \mathrm{hrs}$ of collection.

Enzymes assays- serum AST \&ALT activities were assayed according to the method described in stoeve and makarova. All the reagents used in the work were of anala $\mathrm{R}$ grade.

Data analysis- The difference between the mean serum ALT\&AST level in (alcoholic \&non alcoholic) acute uncomplicated falciparum malaria was analysed using the student's t-test for correlated samples. The difference between the AST/ALT ratio in acute uncomplicated falciparum malaria patients and other diseases were analyzed using the student's $t$ test for independent sample. P- value $<0.05$ were considered significant.

III. Results

Table showing distribution of acute uncomplicated falciparum maiaria under study

\begin{tabular}{|l|c|c|c|}
\hline \multicolumn{1}{|c|}{ Cases } & Males & Females & Total No. \\
\hline Alcoholic & 18 & 10 & 28 \\
\hline Nonalcoholic & 14 & 12 & 26 \\
\hline
\end{tabular}

The study showed 18 males \&10 females in alcoholic groups and 14 males \& 12 females in non-alcoholic groups.Males \& alcoholics were most commonly involved .

Table showing mean \pm SD value of ALT \&AST levels\&AST /ALT ratio in acute uncomplicated falciparum malaria under study.

\begin{tabular}{|c|c|c|}
\hline Enzymes & Alcoholic Cases & Nonalcoholic Cases \\
\hline Alt Level & $51.9 \pm 0.43$ & $41.6 \pm 0.56$ \\
\hline Ast Level & $67.47 \pm 1.19$ & $51.16 \pm 1.37$ \\
\hline Ast/Alt Ratio & 1.3 & 1.23 \\
\hline
\end{tabular}

ALT \& AST activities were raised in 20 patients out of 28 alcoholic patients. ALT \& AST activities were raised only in 8 patients out of 26 non alcoholic patients. Mean ALT \& AST values in both groups of acute uncomplicated falciparum malaria were found to be follows.

Alcoholic patients:- 51.9 \pm 0.43 (ALT), $67.47 \pm 1.19$ (AST)

Non alcoholic patients:- 41.6 \pm 0.56(ALT), $51.16 \pm 1.37$ (AST)

The activities of both ALT \& AST were found to be significantly higher in falciparum malaria patients, $\mathrm{P}<0.05$. Table showing comparative AST/ALT ratios in acute uncomplicated falciparum malaria with some selected chronic liver diseases previously reported. 
A Comparative Study Of Serum ALT \&AST Lvel And AST/ALT Ratio In Alcoholic And ...

\begin{tabular}{|c|c|}
\hline Disease & Ast/Alt Ratio \\
\hline Acute Uncomplicated Falciparum Malaria(Alcoholic) & 1.3 \\
\hline Acute Uncomplicated Falciparum Malaria(Nonalcoholic) & 1.23 \\
\hline A 1 c o & 2.6 \\
\hline Nonalcoholic Steatohepatit is & 0.9 \\
\hline 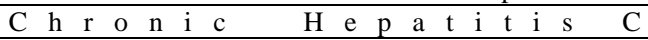 & 0.99 \\
\hline $\begin{array}{lllllllllllllllll}\mathrm{C} & \mathrm{h} & \mathrm{r} & \mathrm{o} & \mathrm{n} & \mathrm{i} & \mathrm{c} & & \mathrm{H} & \mathrm{e} & \mathrm{p} & \mathrm{a} & \mathrm{t} & \mathrm{i} & \mathrm{t} & \mathrm{i} & \mathrm{s} \\
\end{array}$ & 0.8 \\
\hline
\end{tabular}

The AST/ALT ratio of $1.23 \& 1.30$ in falciparum malaria patients is significantly higher than the AST/ALT ratio in other chronic liver diseases except alcoholic liver disease, $\mathrm{P}<0.05$.

\section{Discussion}

The normal healthy values for serum AST \& ALT are in the range of 10-40 U/litre. Falciparum malaria is the leading cause of death $\&$ disability, particularly in tropical countries. The pathogenesis of falciparum malaria involves the hepatic stage where infective sporozoites invade and multiply in the hepatocytes and an erythrocytic stage where merozoites cause the destruction of infected red blood cells prior to their differentiation into male and female gametocytes. Since both the liver and erythrocytes are rich sources of AST \& ALT, Falciparum parasites activities in these organ and tissues can lead to damage of these tissues and consequent release of AST \& ALT and causing increase in their levels. Alcohol also has inflammatory\& necrotic effects on the liver cells, so in alcoholic patients with falciparum malaria AST, ALT level can be high and these patients should be treated aggressively. High AST/ALT ratio in alcoholic liver disease is due to damaging effect of alcohol on centriportal liver cells which are rich in AST and low serum activities of ALT. The comparatively low AST/ALT ratio in other diseases can be explained by difference in etiology of these diseases. So considering earlier reports which indicates that an AST/ALT ratio > 1 is unique and indicative of alcoholic liver disease or cirrhosis, the findings of an AST/ALT ratio $>1$ in acute falciparum malaria is a justification that changes in AST/ALT ratio in other diseases should be interpreted with caution particularly in malaria endemic zones where AST/ALT ratio could be indicative of falciparum malaria and not alcoholic liver disease or cirrhosis.

\section{Conclusion}

This study showed mean serum ALT \& AST levels were significantly increased in both alcoholic \& nonalcoholic acute uncomplicated Falciparum malaria patients, but the levels were increased more in alcoholic patients signifying more hepatic dysfunction and so to start the adequate and aggressive management at the earliest to prevent the further complications. This study also showed AST/ALT Ratio>1 can be indicative of Falciparum Malaria,contradicting earlier reports that AST/ALT Ratio $>1$ is unique and indicative of alcoholic liver disease or cirrhosis oniy. This study is not the final verdict. More future studies will either corroborate or contradict this study.

\footnotetext{
References

[1]. Harrison's principle of internal medicine, $18^{\text {th }}$ edition, $1688-1710$

[2]. Grostadt M.Rej Mitochondrial aspartate aminotransferase determined by fast protein liquid chromatography 1980;36: 348-350

[3]. Catbreath D F. clinical chemistry. Philadelphia W.B Sanders Company 1992; 468p

[4]. Huncrantz R. Galuman Liver investigation in 149 asymptomatic patients with moderately elevated activities of serum aminotransferases 1986; 21: 109-113

[5]. Rosentnal P, Hasgut M aminotransferase as a prognostic index in patients with liver disease. Clin chem. 1989; 36: 346-348

[6]. Sorbi D, Boynton $\mathrm{j}$, the ratio of AST to ALT : potential value in differentiating non alcoholic steatohepatitis from alcoholic liver disease.

[7]. Reddy DW, LOOAT, Levine RA AST/ALT ratio $>1$ is not diagnostic of cirrhosis in patients with chronic hepatitis C.

[8]. Miller LH, Baruch DI, Marsh k, Docunbo k, The pathogenic basis of malaria, Natur 2002; 415: 673-679.
} 